\title{
Distribution of Acoustic Emission Avalanches in Martensitic Transformations
}

\author{
E. Vives, J. Ortín, Ll. Mañosa, I. Ràfols and A. Planes \\ Departament d'Estructura i Constituents de la Matèria, Facultat de Física, Universitat de Barcelona, \\ Diagonal 647, 08028 Barcelona, Catalonia, Spain
}

\begin{abstract}
Measurements of the Acoustic Emission generated during a Martensitic Transformation are presented. Statistical analysis of the amplitude and lifetime of signals has revealed power-law distributions for both magnitudes. The critical exponents of these distributions have been evaluated. The results are discussed in terms of modern theories of avalanche dynamics for first order phase transitions.
\end{abstract}

\section{INTRODUCTION}

In thermoelastic Martensitic Transformations (MT), as temperature is decreased, the system evolves step by step through a succession of metastable states. This behaviour stems from the fact that the nucleation and growth of domains of the low temperature phase modifies the internal strain field of the system due to the significant shape change of the unit cell [1]. Strain energy is then elastically stored in the lattice (the transition is called thermoelastic for this reason) and blocks subsequent growth of the new phase completely, leaving the system in a metastable two-phase state. Additional undercooling is then needed for the transition to proceed. As a consequence, the transition is athermal: temperature acts as an external field and thermal fluctuations do not play a relevant role. Evolution from one metastable state to another takes place by avalanches, with associated energy dissipation [2]. Part of this energy has a reversible character: this is the latent heat associated with the fraction of transformed material. The remaining part is essentially irreversible and is responsible for the hysteresis observed in such kind of transitions. The source of this irreversible energy is mainly the relaxation of elastic energy, released as elastic waves, usually in the ultrasonic range. This is the so-called Acoustic Emission (AE) which can be detected with appropriate transducers [3]. This AE has many similarities with seismic waves generated during an earthquake. In both cases the main underlying physical mechanism generating the elastic waves is a shear. The important point is that AE signals contain information on both the size and lifetime of the avalanches occurring during the transition [4].

Recently there has been an increasing interest in the study of externally driven systems, with spatial and temporal degrees of freedom, that spontaneously self-organize into a stationary state far from equilibrium [5]. In this state the system displays avalanches of all sizes that give rise to power-law spatial and temporal correlation functions, characteristic of a critical state. This behaviour has been called self-organized criticality. The idea has been applied to earthquakes, where the well known Gutenberg-Richter law for their magnitude distribution can be understood as a result that the crust of the Earth is being driven to the critical state by the tectonic plate motion [6].

In view of the existing similarities, it has been suggested that systems undergoing thermoelastic MT are good candidates to behave in a self-organized critical way. With this idea in mind, we have detected and statistically analyzed a large number of AE signals generated during the forward and reverse $\mathrm{MT}$ in a $\mathrm{Cu}$ - 
$\mathrm{Zn}-\mathrm{Al}$ shape memory alloy.

The paper is organized as follows. In section 2 we describe the experimental set-up. In section 3 we present the results obtained and finally in section 4 we discuss the results in terms of the self-organized criticality theory and more recent ideas proposed to explain avalanche dynamics during first order phase transitions.

\section{EXPERIMENTAL}

A single crystal grown by the Bridgman method from elements of purity $99.99 \%$, with composition $\mathrm{Cu}-$ $17.0 \% \mathrm{Zn}-13.7 \mathrm{at} \% \mathrm{Al}$, has been investigated. A disc shaped specimen of $12.6 \mathrm{~mm}$ diameter and $1.9 \mathrm{~mm}$ thickness has been cut from the original crystal using a low speed diamond saw and the surfaces have been mechanically polished. On cooling the transition starts at $M_{s} \approx 299 \mathrm{~K}$ and finishes at $M_{\mathrm{f}} \approx 268 \mathrm{~K}$. The corresponding temperatures on heating are $A_{s} \approx 278 \mathrm{~K}$ and $A_{f} \approx 308 \mathrm{~K}$. We have performed several heating and cooling runs before starting our measurements in order to ensure a very good reproducibility of the transformation.

The sample is placed on the surface of a copper block which is heated and cooled by means of a thermobattery using the Peltier effect. The temperature of the block, which is measured with a four wire Pt-100 resistance, is computer controlled with an accuracy better than $0.1 \mathrm{~K}$. The temperature of the sample is assumed to be the same as the temperature of the copper block.

AE emission signals are detected by a resonant piezoelectric transducer acoustically coupled to the surface of the sample. The electrical signal from the transducer is amplified and filtered in the frequency range $400 \mathrm{kHz}-2 \mathrm{MHz}$ by a Bruel \& Kjaer preamplifier and amplifier with a variable overall amplification ranging from 40 to $100 \mathrm{~dB}$. The output of the amplifier provides a DC peak with amplitude equal to the positive peak level of the signal. It has a short rise time $(0.2 \mu \mathrm{s})$ and a time constant of $16 \mu \mathrm{s}$. Blocks of 1000 signals are recorded using a fast transient recorder which digitizes signals whose amplitude exceeds a given trigger level above the background noise, at a sampling frequency of $0.5 \mathrm{MHz}$. For each signal its maximum amplitude and lifetime are determined (see fig. 1). All experiments have been performed at cooling/heating rates of around $1 \mathrm{~K} / \mathrm{min}$. Moreover we have checked that the results are insensitive to changes of this magnitude, at least in the range $0.1-3 \mathrm{~K} / \mathrm{min}$.

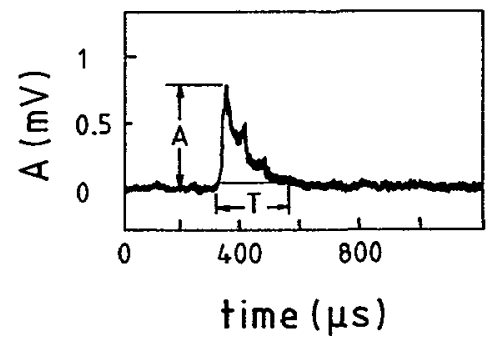

Fig. 1 Typical AE signal detected with a piezoelectric transducer. A and $\mathrm{T}$ are the amplitude and lifetime corresponding to this signal.

\section{RESULTS}

In fig. 2 we show the joint distribution of amplitudes (A) and lifetimes (T) obtained with the whole set of recorded signals (during heating and cooling runs). The figure also shows a projection on the A-T plane in $\log -\log$ representation. This plot suggests that statatistically, $\mathbf{A} \sim \mathbf{T}^{\mathbf{x}}$. A rough estimation of the exponent $\mathrm{x}$ has been obtained by fitting a straight line to the data in the $\log -\log$ plot. The value obtained is given in table $I$.

The marginal amplitude and lifetime distributions are represented in $\log -\log$ plots in figs. 3a and $3 b$ 
respectively. In both figures we have separated data obtained in the heating and cooling runs. In all cases power law regimes (N(A) $\sim \mathrm{A}^{-\alpha}$ and $\mathrm{N}(\mathrm{T}) \sim \mathrm{T}^{-r}$ ) corresponding to a linear behaviour in the $\log -\log$ plots, are obtained. The exponents characterizing the distributions have been evaluated by least square linear fits. The resulting values are also given in table $I$.

At low amplitudes, the breakdown of the power law is due to the detection threshold. At high amplitudes the distribution is influenced not only by the amplifier cutoff but also by possible finite size effects. The lifetime distributions are less accurate than the amplitude ones: as is clearly seen in the A-T projection of the joint distribution (see fig.2), for small durations $\left(T<810^{-5} \mathrm{~s}\right)$ the number of signals detected for a given time is strongly affected by the amplitude detection threshold; moreover, the resonant behaviour of the transducer may have an influence on the distributions (mainly at short times). In order to estimate this effect we have performed some measurements with transducers of different resonant characteristics and conclude that reliable data are obtained for times longer than $25 \mu \mathrm{s}$. For $\mathrm{N}(\mathrm{A}) \sim \mathrm{A}^{-\alpha}, \mathrm{N}(\mathrm{T}) \sim \mathrm{T}^{-\tau}$ and $\mathrm{A} \sim \mathrm{T}^{\mathrm{x}}$, it is easy to show that the following simple relation between the exponents: $x=(\tau-1) /(\alpha-1)$ must hold. In order to check this relation, we have included in table 1 , together with the fitted value of $x$, its value calculated from the former equation and using the values of $\alpha$ and $\tau$. The values obtained from the two methods are in very good agreement.

\begin{tabular}{|c|c|c|c|c|}
\hline Exponent & $\mathrm{x}$ & $\alpha$ & $\tau$ & $(\tau-1) /(\alpha-1)$ \\
\hline & $0.9 \pm 0.4$ & $3.6 \pm 0.8$ & $3.4 \pm 0.8$ & $0.9 \pm 0.5$ \\
\hline
\end{tabular}

Table I. Fitted values of the exponents $\mathrm{x}, \alpha$ and $\tau$.
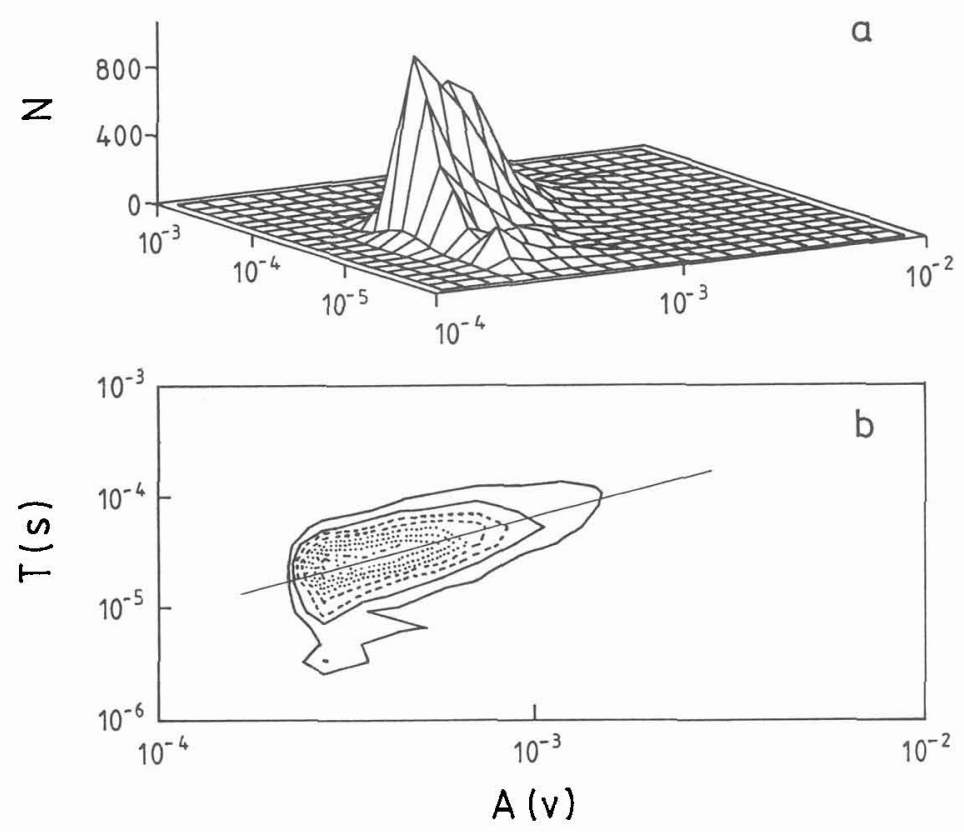

Fig. 2 (a) Joint distribution function of amplitudes (A) and lifetime (T). (b) Projection on the A-T plane in $\log -\log$ representation. The straight line is a linear fit to the data from which a rough estimation of the exponent $\mathrm{x}$ is obtained. 

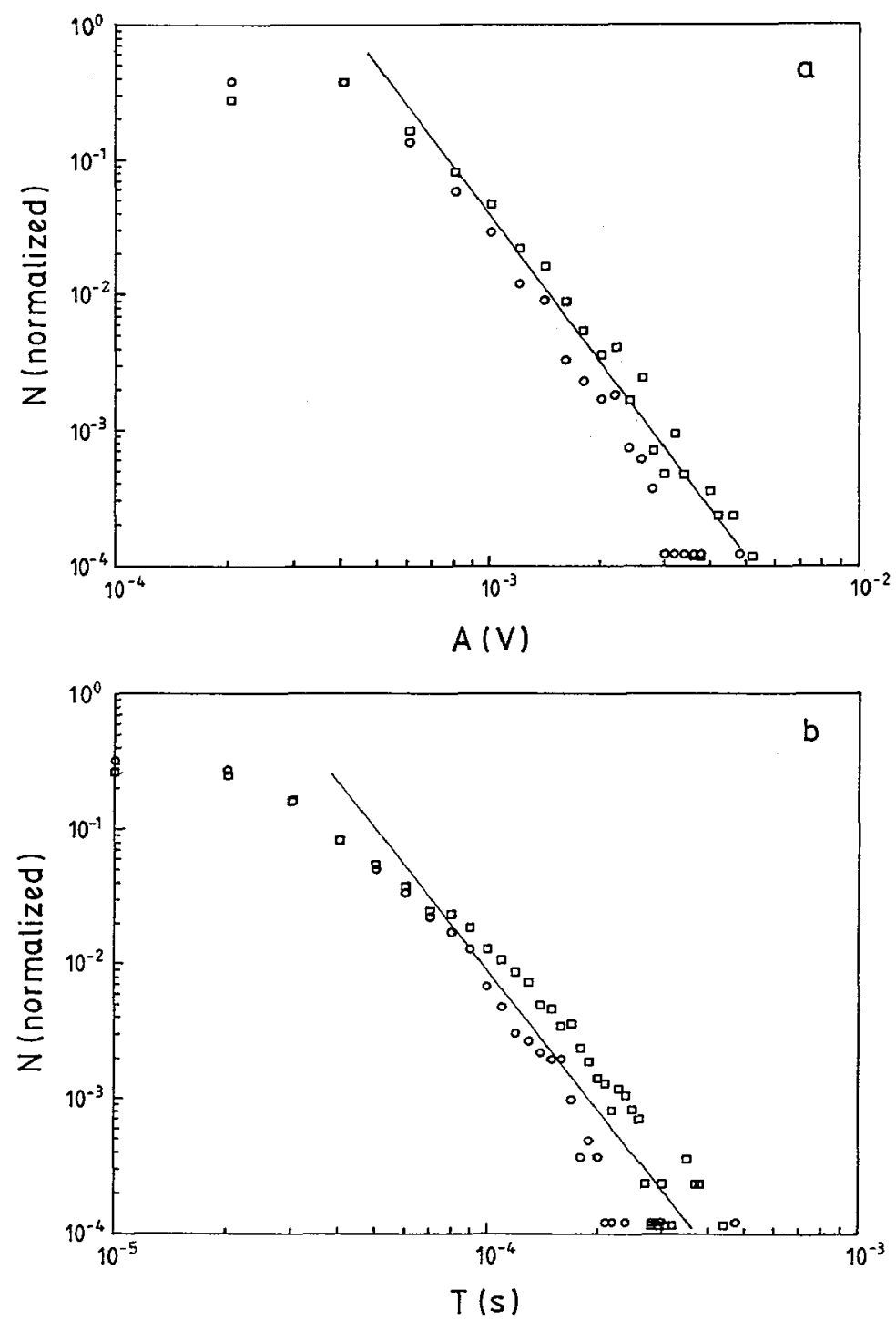

Fig 3 Log-log plot of the marginal amplitude (a) and lifetime (b) distributions of AE signals recorded during heating $(\square)$ and cooling $(O)$ runs. The lines are best fits in the linear regions.

An important aspect to be investigated is whether or not the distributions of amplitudes and durations of AE signals captured during small temperature intervals $\Delta T$ (but large enough to record a meaningful statistical set of AE signals) in the cooling and heating processes are characterized by the same exponents than the distributions obtained with the signals recorded in the whole temperature range of transformation. In fig. 4 we show the amplitude distributions corresponding to temperature intervals centered at different temperatures during the cooling process. Within the experimental uncertainties, our results indicate that the fitted exponents are independent of the temperature range at which the signals have been captured. Nevertheless it is worth noticing that the breakdown of the power-law at high amplitudes depends on the transformation range considered. For $\Delta \mathrm{T}$ at the beginning of the transformation the power-law behaviour is satisfied up to higher amplitudes. This may be due to the fact that in this temperature range the fraction of martensite in the sample is small and the transition occurs in a nearly free way. As a consequence finite size effects are less significant in this region. 


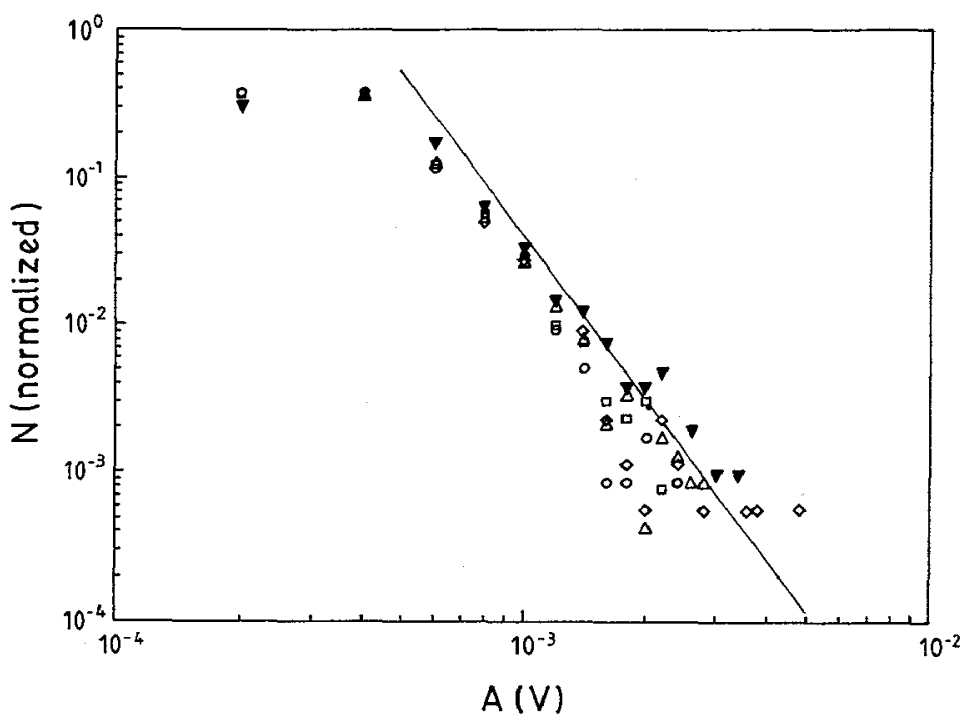

Fig. 4 Log-log plot of the amplitude distribution of $\mathrm{AE}$ signals recorded in small temperature intervals $\Delta \mathrm{T}$ during cooling around: $(0)-5^{\circ} \mathrm{C},(\square) 0^{\circ} \mathrm{C},(\diamond) 5^{\circ} \mathrm{C},(\Delta) 10^{\circ} \mathrm{C},(v) 15^{\circ} \mathrm{C}$. The straight line has the same slope than the one in fig. $2 a$.

\section{DISCUSSION}

The results obtained in our statistical study of the AE generated during the MT of a Cu-Zn-Al alloy suggest that the behaviour of thermoelastic systems during the growth process can be explained in the framework of the SOC theory. Indeed the SOC theory has already been invoked to explain the power-law distribution of avalanche size and duration in other first order phase transitions where, as occurs in MT, the role of thermal fluctuations is not relevant. This is for example the case of the field-induced changes of magnetization in ferromagnetic materials (Barkhausen effect) [7]. However this interpretation has been recently criticized by Sornette [8], who indicates that this kind of processes, associated to first order phase transitions, cannot operate persistently at a stationary critical state, as required by the SOC theory. He noted that these processes are better described by the sweeping of a control parameter (that is, the field that induces the transition) which is responsible for the system to evolve along the two-phase coexistence region. Then, the observed power law distributions could be better interpreted as stemming from fluctuations associated to the magnitude conjugated to the field spanning the phase diagram. It is important to note that these fluctuations have not a thermal origin. For instance, they can be understood as coming from the existence of intrinsic disorder in the system. In the case of MT the disorder arises from defects (which are crucial for the nucleation of the martensitic phase) and from the intrincate character of the internal stresses generated by the transformation itself.

With this idea in mind, Sethna et al. [9] have studied the evolution of the magnetization during a field induced first order transition in a random field Ising model at $0 \mathrm{~K}$ (i.e. neglecting thermal fluctuations). They qualitatively reproduced the specific memory features of the hysteresis loops and subloops observed in $\mathrm{MT}$, and obtained that, for a given amount of disorder, sweeping of the magnetic field produces avalanches of the magnetization with no characteristic length scale, showing a power-law size distribution. For different amounts of disorder the distribution appears as a power-law multiplied by an exponential prefactor. More recently, it also been shown that a spin glass model is also suitable to reproduce this kind of behaviour [10]. Here the disorder is not introduced as a static random field but rather on the spin-spin interactions, mimicking the complex elastic interactions between transformed and non-transformed domains. 
Two aspects of these models are important to be discussed in relation to our experimental results. The first one is the fact that the critical behaviour (pure power-law distributions) is only obtained after fine tuning of the amount of disorder. This point suggests the following question: why the real system appears to stay at the critical amount of disorder? We provide two possible answers to that question: (1) The experimental uncertainties make difficult to detect the possible existence of an exponential prefactor. (2) A more subtle explanation could be that internal relaxation during the growth process spontaneously modifies the disorder present in the system until the critical state is reached. This idea is supported by the fact that the growth process appears to be independent of a particular heat treatment after a number of cycles (in our experiment the sample has been initially cycled until a reproducible state has been reached).

The second aspect deals with the fact that in the models, for the critical amount of disorder, the critical state is obtained at a given value of the induction field (critical field). Away from this value of the field, the size of avalanches shows a power law distribution but the corresponding exponent appears to be dependent on the value of the field. This is contrary to what is observed in our experiments where the exponent is independent of temperature, i.e. of the driving field. This result makes the growth process in MT to compare more favourably to the predictions of the SOC theory.

To conclude we have shown the absence of intrinsic scales in the dynamics of MT. The effect of intrinsic disorder in the system seems a crucial ingredient to explain such behaviour. However up to now there is not a complete theory able to explain it in a satisfactory way.

\section{Acknowledgments}

This work has been supported by CICyT, project nr. MAT92-884.

\section{References}

[1] A.G. Kachaturyan, Theory of Structural Transformations in Solids (Wiley, New York, 1983)

[2] A. Planes, J.L. Macqueron, M. Morin, G. Guénin, phys. stat. sol (a) 66 (1981) 717

[3] J. Baram, M. Rosen, Acta metall., 30 (1982) 655; L1. Mañosa, A.Planes, D. Rouby, J.L. Macqueron, Acta metall. mater. 38 (1990) 1635

[4] E. Vives, J. Ortín, L1. Mañosa, I. Ràfols, R. Pérez-Magrané, A.Planes, Phys. Rev. Lett., 72 (1994) 1694

[5] P. Bak, C. Tang, K. Wiesenfeld, Phys. Rev. Lett., 59 (1987) 381

[6] Z. Olami, H.J.S. Feder, K. Christainsen, Phys. Rev. Lett., 68 (1992) 1244

[7] P.J. Cote, L.V. Meisel, Phys. Rev. Lett., 67 (1991) 1334; L.V. Meisel, P.J. Cote, Phys. Rev. B, 46 (1992) 10822

[8] D. Sornette, J. Phys. I (France), 4 (1994) 209

[9] J.P. Sethna, K. Dahmen, S. Kartha, J.A. Krumhansl, B.W. Roberts, J.D. Shore, Phys. Rev. Lett., 70 (1993) 3347

[10] E. Vives, A.Planes, Phys. Rev. B, 50 (1994) -- 